\title{
Surveillance of Drug-Resistant Tuberculosis in Spain (2001-2015)
}

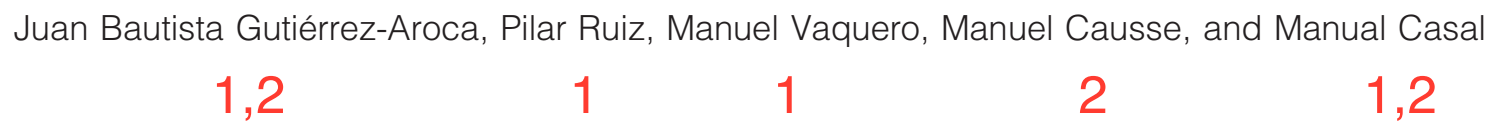

Multidrug-resistant (MDR) tuberculosis (Tb) Is a cause of increasing concern. This study investigated first-line anti-TB drug resistance in Mycobacterium tuberculosis strains submitted to the Tuberculosis Reference Center in Córdoba (Spain) between 2001 and 2015. A total of 1,207 cultures were tested against first-line drugs using the BACTEC MGIT 960 system. Resistance to first-line drugs was detected in 207 strains (17.2\%), the greatest resistance being found in INH $(5.3 \%)$ followed by streptomucin $(3 \%)$, pyrazinamide $(2.2 \%)$, rifampicin $(1 \%)$, and ethambutol (0.2\%). A total of $1.9 \%$ of strains were MDR_s strains displayed resistance to four drugs, and three strains to five drugs. In view of resistance observed, careful surveillance of drug resistance is recommended.

Keywords: tuberculosis, resistance, mycobacterium

\section{Introduction}

$\mathbf{T}$ UBERCULOSIS (TB) REMAINS A major global health problem, and multidrug-resistant (MDR) TB is a cause of increasing concern. In 2016, according to the World Health Organization (WHO), an estimated 10.4 million new cases of TB were notified, and an estimated 1.3 million people died of the disease, which remained one of the top 10 causes of death worldwide, ranking above HIV infection and malaria. ${ }^{1}$

MDR-TB continues to constitute a major public health crisis, affecting an estimated 53 million people. The WHO reported 490.00 new cases of MDR-TB in $2016{ }^{1}$

MDR in TB is by no means a new phenomenon; its incidence has varied over the years, depending on the drugs used to treat the disease.

As early as the 1950s, when the first specific anti-TB drugs were introduced, resistance soon developed to streptomycin when used alone. ${ }^{2}$ To tackle this problem, combined treatments were developed, associating streptomycin with para-aminosalycilic acid and isoniazid (INH); this combined therapy, used over a period of 18-24 months, cured most treatment-naive patients whose compliance was ensured by admission to hospitals or sanatoria.

In the course of the 1960s, the success of this therapy prompted a degree of overconfidence; rules were relaxed, and treatments started to be administered on an outpatient basis with insufficient supervision. This led to the emergence of resistance to INH, which proved problematic in some regions, where patients displayed resistance to both streptomycin and INH. ${ }^{3}$ These early cases of MDR-TB could then infect others, who developed initial drug-resistant TB. This contagion prompted an increase in the number of patients with chronic disease, who-though not dying-increased the incidence of environmental drug resistance. ${ }^{4}$

In the 1970s, the situation was alleviated by the introduction of rifampicin and pyrazinamide; closely supervised short-term combined therapy (6-9 months) succeeded in curing cases of initial INH-resistant TB..$^{5,6}$

By the 1980s, excessive confidence in the theoretical efficacy of existing therapies-coupled with a lack of resources, insufficient healthcare, and other factors including emigration, AIDS, and drug addiction-prompted the emergence of resistance not only to rifampicin but also, more worryingly in health terms, to both INH and rifampicin, the two drugs most widely used to treat $\mathrm{TB} .^{7}$ It was at this point that the term MDR was coined. ${ }^{8}$ In 2014, an estimated 480,000 people developed MDR-TB worldwide. Reports suggest that MDR-TB currently accounts for over $1.2 \%$ of every 10,000 cases of TB, and over $4 \%$ of all new cases, causing around 150,000 deaths per year, of which the WHO estimates that only around $7 \%$, are diagnosed. ${ }^{1}$

The present study reports on resistance to first-line antiTB drugs in Mycobacterium tuberculosis strains studied between 2001 and 2015 at the Cordoba Tuberculosis Reference Centre (Spain). The Reference Center receives samples from Andalusia, with a population of 8,379,880; and the proportion of samples with respect to the total number of samples in Spain represents a percentage of $18 \%$.

\footnotetext{
AU3 Facultad de Medicina, Centro de Referencia para el Control de la Tuberculosis, Servicio de Microbiología Hospital Universitario Reina 1 Faculty of of Rédoba, Spain. 2Tuberculosis control Reference Center. Microbiology Serviqe. Universitary Hospital "Reina Sofia" Cordoba, Spain.
} 


\section{Materials and Methods}

\section{Study design}

This cross-sectional study sought to investigate drug resistance in $M$. tuberculosis isolates recovered from culturepositive cases diagnosed at the Cordoba Tuberculosis Reference Centre (Spain) between 2001 and 2015. The study was conducted in accordance with international recommendations for drug-resistance surveillance.

Over the 15-year period under study, a total of 56,708 clinical specimens from patients with suspected TB were processed. M. tuberculosis was detected in 1,207 cultures from 1,207 patients, all of whom were tested for resistance to first-line anti-TB drugs: streptomycin, isoniazid, ethambutol, rifampicin, and pyrazinamide.

Cultures were viewed under a fluorescent microscope, decontaminated using a NaCl-NaOH solution, and inoculated into Bactec MGIT 960 TB tubes (Becton-Dickinson, Sparks) containing Middlebrook $7 \mathrm{H} 9$ liquid broth medium supplemented with PANTA (polymyxin B, amphotericin B, nalidixic acid, trimethoprim, and azlocillin) and OADC (oleic acid, albumin, dextrose, and catalase). Cultures were also grown on Lowenstein-Jensen and Coletsos media. Positive cultures were confirmed by Ziehl-Neelsen staining, and $M$. tuberculosis was identified first using the AccuProbe rRNA hybridization assay (San Diego, Califaria). ${ }^{9}$ All $M$. tuberculosis cultures were tested for drug $P$ ance, using ESPII system (Trek Diagnostic Systems, Westlake, Ohio). ${ }^{10}$

When the polymerase chain reaction wintroduced, cultures were identified using the GenoType 2 ycobacterium assays: CM (for 20 common mycobacterial species) and AS (for 17 additional species). ${ }^{11}$ Both assays are based on DNA strip technology involving multiplex amplification with biotinylated primers and reverse hybridization.

All cultures were tested for sensitivity to streptomycin (SM), rifampicin (RIF), ethambutol (EB), isoniazid (INH) and pyrazinamide (PZA), following the MGIT 960 protoco all in the form of lyophilized powder in vials.

Once reconstituted, $0.1 \mathrm{ml}$ of each drug was added to vials containing Middlebrook 7H12 medium; the strain grown on Lowenstein-Jensen medium was then inoculated. Vials were incubated at $37^{\circ} \mathrm{C}$ and read daily until growth was sufficient to enable interpretation.

\section{Statistical analyses}

Epi Info v.7.2 software (Centers for Disease Control, Atlanta, GA) was used for data input and for statistical analyses, which took into account study design. The standard error (SE) was adjusted.

Where there were four or fewer drug-resistant cases, the exact binomial confidence interval was used, without reference to sample weights and without adjusting SE. For statistical analysis, Pearson $\chi^{2}$ test, $p=0.05$ was used as the cutoff for statistical significance.

\section{Results}

All $M$ tuberculosis strains $(1,207)$ were tested for firstline dru 2 istance. A total of 1,000 strains $(82.8 \%)$ were sensitive to all the drugs, while $207(17.2 \%)$ were resistant to one or more drugs (95\%CI 14.9-19.3). Overall resistance
Table 1. Resistance to First-Line Drugs (2001-2015)

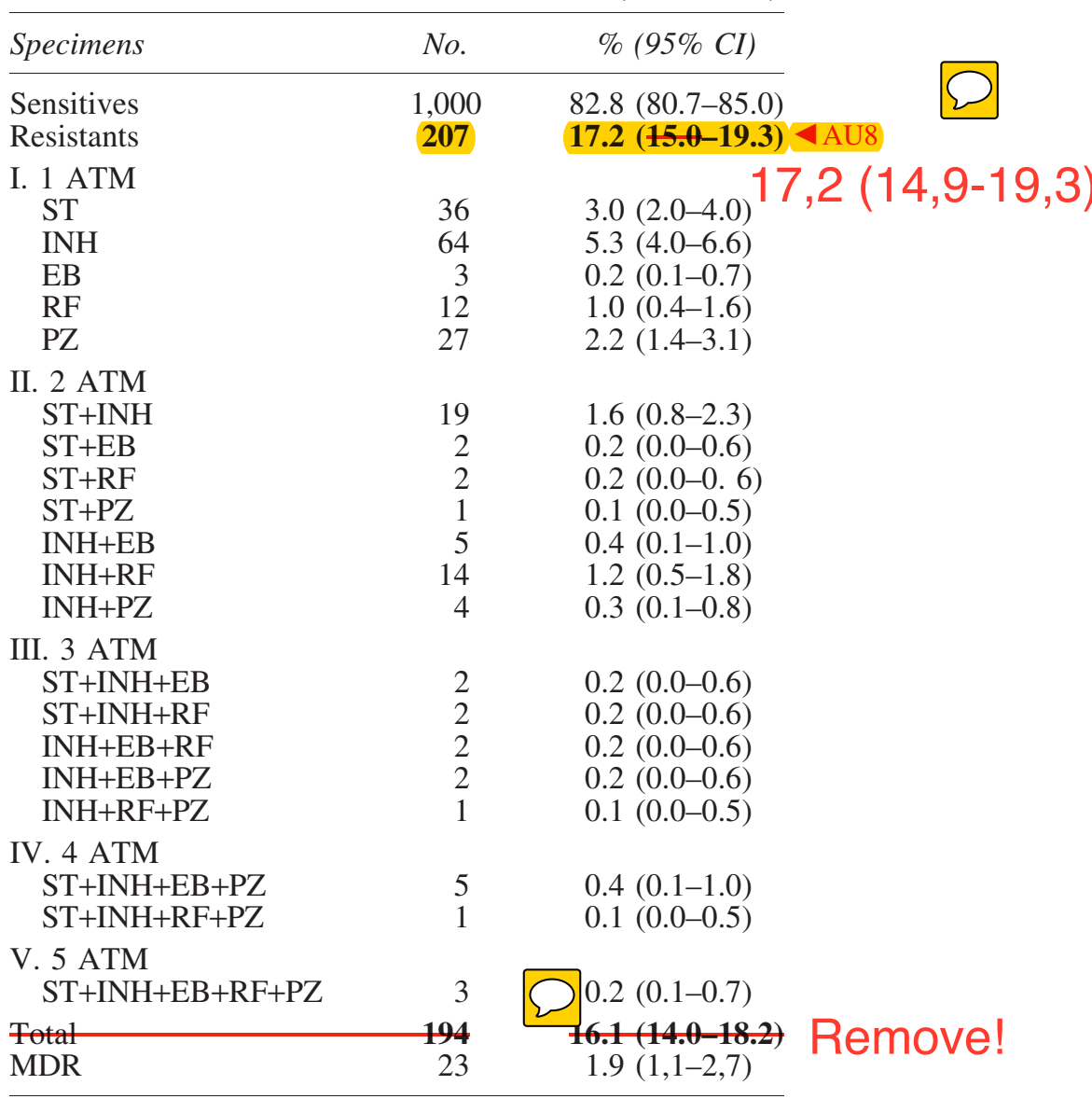

Cordoba Mycobacterial Reference Center.

ST, streptomicin; INH, isoniazide; EB, etambutol; RF, rifampicin; PZ, pirazinamide; MDR, multidrug-resistant.

The most widespread resistance was recorded for isoniazid (5.3\% of all resistant strains; 95\% C I 4.0-6.6), followed by streptomycin $(3 \%$; 95\% CI $2.0-4.0)$, pyrazinamide $(2.2 \%$; $95 \%$ CI $1.4-3.1)$, rifampicin (1\%; 95\% CI $0.4-1.6)$, and finally ethambutol $(0.2 \%$; $95 \%$ CI $0.1-0.7)$.

Among strains resistant to more than one drug, the greatest resistance was recorded for streptomycin+isoniazid (1.6\%; 95\%CI $0.8-2.3)$, followed by streptomycin+ rifampicin $(1.2 \%$; $95 \% \mathrm{CI} 0.5-1.8)$; MDR strains resistant to other combinations accounted for far less than $1 \%(95 \% \mathrm{CI}$ $0.1-1.0)$. Only six strains $(0.5 \%$; $95 \%$ CI $0.1-0.9)$ were resistant to streptomycin, isoniazid, ethambutol, and rifampicin, while three strains were resistant to all five drugs tested (0.4\%; 95\%CI 0.1-1.0).

A total of $1.9 \%(95 \%$ CI $1.0-2.8)$ of resistant strains displayed multi-resistance to isoniazid+rifampicin. However, resistance rates varied considerably over the 15 -year study period, ranging from $7.6 \%$ (95\% CI 5.9-9.3) in 2006, to 5.1\% (95\%CI 3.7-6.5) in 2005 and 3\% (95\%CI 1.9-4.1) in 2002; in other years, resistance rates approached the average, or no MDR strain was detected (2001, 2010, 2013, 2014).

\section{Discussion}

The overall resistance rates obtained here $(11.8 \%)$ are higher than those reported for centers elsewhere in the Iberian Peninsula (Table 2), with the exception of those $<\mathrm{T} 2$ 
Table 2. Resistance to First-Line Drugs (2001-2015)

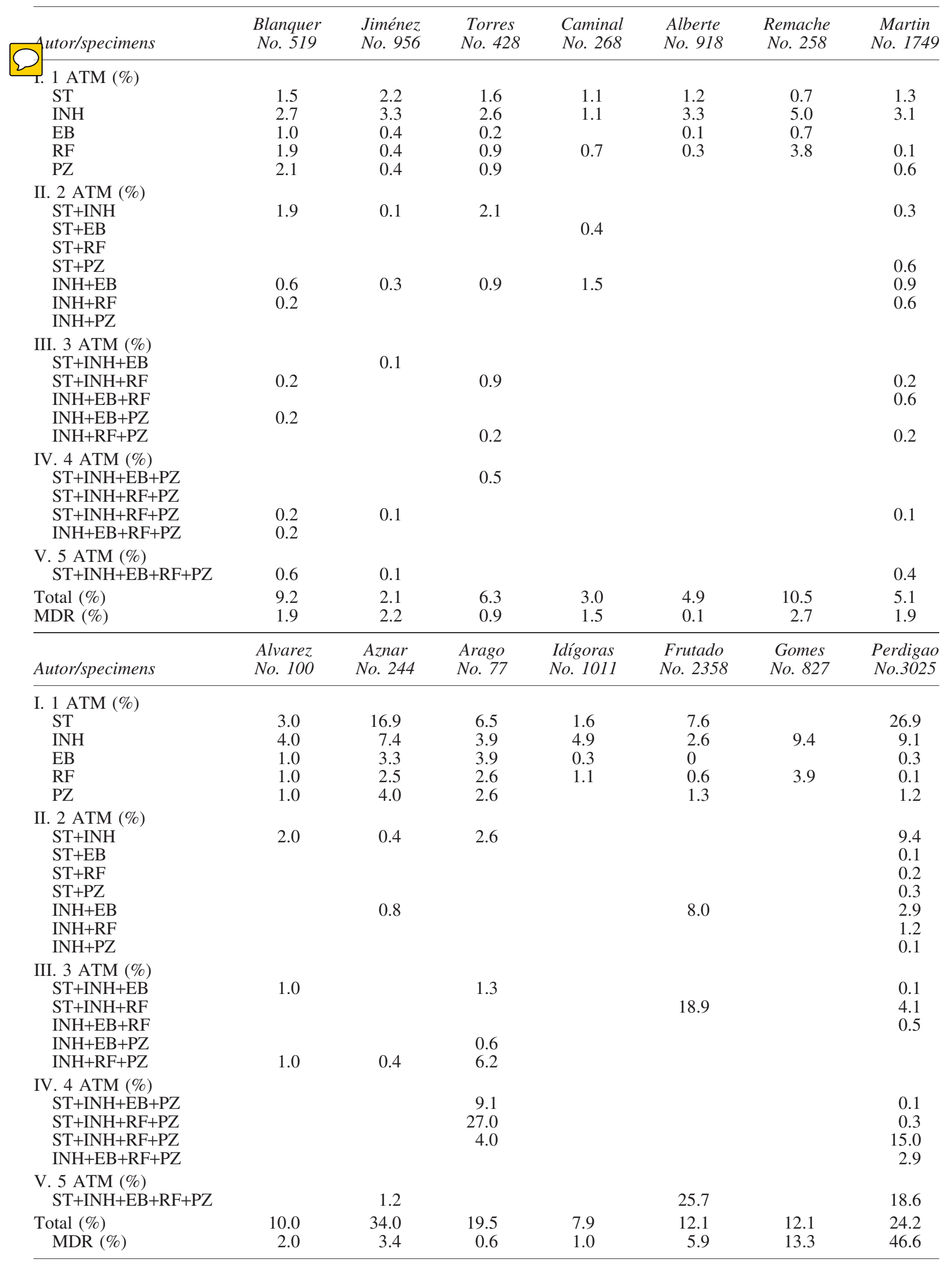

Centers consulted. 
reported by Aznar for Madrid (34\%) and by Aragó for Sagunto $(19.5 \%){ }^{13,14}$

The multi-resistance rate recorded here $(1.9 \%)$ is lower than that reported by the authors themselves in an earlier study for the same area (5.8\%), and considerably lower than the $34.0 \%, 10.5 \%$, and $10 \%$ reported by Aznar (Madrid), Remacha (León), and Alvarez (Galicia), respectively. ${ }^{13,15,16}$

With regard to findings for individual anti-TB drugs, resistance to streptomycin $(3.0 \%)$ was higher than that reported by all except Aznar (16.9\%) and Aragó (6.5\%); other authors noted rates similar to those obtained here. ${ }^{17-25} \mathrm{Re}$ sistance to isoniazid (5.3\%) exceeded that recorded by any other author except Aznar (7.4\%), while the ethambutol resistance rate $(0.2 \%)$ was lower than those obtained by all except Aragó (3.9\%) and Aznar (3.3\%). Resistance to rifampicin $(1.0 \%)$ was also lower than that reported in the literature for Spain; Remacha obtained rates of 3.8\%, Aragó $2.6 \%$, and Aznar 2.5\%. By contrast, resistance to pyrazinamide $(2.2 \%)$ was higher than that reported by all authors except Aznar (4.0\%) and Aragó (2.6\%). Comnaring our results to those published by WHO (Globa' Cam whereas it is maintained in Spain.

In terms of resistance to two or more most striking finding was for the ST (1.6\% resistance); highu figures are Aragó, ${ }^{14}$ Torres (Zaragoza), ${ }^{2}$ Alvarez, $(2.6 \%, 2.1 \%, 2.0 \%$, and $1.9 \%$, renect $\sim 1 y)$; all onter authors report lower resistance rates. Resistance to the INH+RF combination (1.2\%) was comparable only to that noted by Caminal 27 (5), lower rates being reported by all other centers. Resistance to other drug combinations tested ( $1 \%$ or lower) was relatively negligible.

Overall resistance findings were considerably lower than in Portugal (Table 2): Pertigao, for example, reported overall resistance of $24.16 \%$, while Furtado recorded rates of $12.13 \% .^{29,30}$

Multi-resistance rates also appear to be much higher in Portugal; Perdigao, Furtado, and Gomes report rates of $46.6 \%, 5.9 \%$, and $13.3 \%$, respectively. ${ }^{29-31}$

Recorded rates of resistance to individual anti-TB drugs in Portugal are in some cases higher than those obtained here: Perdigao, for example, reported $26.9 \%$ resistance to streptomycin and $9.1 \%$ resistance to isoniazid; findings for other drugs, however, were similar to those recorded here. ${ }^{32-34}$

It should be noted that resistance rates in our study were generally lower than those recorded in previous studies by the same authors. This may be due to earlier and swifter disease diagnosis, and improved clinical attention; constant patient surveillance ensures better compliance with combined drug therapies.

Even so, the findings reported here suggest that MDR, a phenomenon that emerged in the 1950s in response to early anti-TB drugs, continues to represent a challenge in the $21 \mathrm{st}$ century.

Swifter and more precise microbiological diagnosisbased on improved systems able to cut diagnosis times from days or weeks to a matter of hours-is essential to combat MDR-TB; the development of new, more efficacious, drugs would help to limit cross-resistance and could reduce treatment time from months to days. All this should be underpinned by the fastest-possible sensitivity testing. ${ }^{35}$
Although resistance rates and MDR-TB prevalence remain relatively low, constant surveillance of MDR is essential to adequate health control.

\section{Acknowledgments}

This work was supported by the Spanish Ministry of Health and Consumer Affairs, Instituto de Salud Carlos IIIERDF, Spanish Network for Research into Infectious Diseases (REIPI RD06/0008).

\section{Disclosure Statement}

No competing financial interests exist.

\section{References}

1. World Health Organization. 2017.Global Tuberculosis Report 2017. WHO, Geneva.

2. Youmans, G.P., E.H. Williston, W.H. Feldman, and H.C. Hinshaw.1946. Increase in resistance of tubercle bacilli to streptomycin; a preliminary report. Proc. Staff Meet. Mayo lin. 21:126-127.

dani, A. 1976. Short-course (6-month) treatment of lmonary tuberculosis (Second East African/British Medical Research Council Study). Bull. Int. Union Tuberc. 51:53-56.

4. Pearson, M.L., J.A. Jorep, and T.R. Frieden. 1970. Nosocomial transmission of multidrug-resistant tuberculosis. $\mathrm{N}$. Engl. J. Med. 283:191-196.

5. Edlin, B., J.L. Tokars, and H. Crieco. 1992. An outbreak of multidrug-resistant tuberculosis among hospitalised patients with the acquired immunodeficiency syndrome. $\mathrm{N}$. Engl. J. Med. 326:514-1521.

6. Fischl, M.A., R.B. Uttamchardami, and G.L. Daikos. 1992. An ourbreak of TB caused by multidrug-resistant tubercle bacilli among patients with HIV infection. Ann. Intern. Med. 117:177-183.

7. Casal, M, M.Vaquero, H. Rinder, E. Tortoli, J.Grosset, S. Rusch-Gerdes, J.B. Gutiérrez, and V.A. Jarlier. 2005 Case-control study for multidrug-resistant tuberculosis risk factors in four European countries. Microb. Drug Resist. 11:61-65.

8. Jacobs, R.F. 1994. Multiple DR tuberculosis. Clin. Infect. Dis. 19:1-10.

9. Reisner, B.S., A.M. Gatson, and G.L. Woods. 1994. Use of Gen-Probe AccuProbes to identify Mycobacterium avium complex, Mycobacterium tuberculosis complex, Mycobacterium kansasii, and Mycobacterium gordonae directly from BACTEC TB broth cultures. J. Clin. Microbiol. 32: 2995-2998.

10. Ruiz, P., F.J. Zerolo, and M. Casal. 2000. Comparison of susceptibility testing of Mycobacterium tuberculosis using the ESP culture system II with that using the BACTEC method. J. Clin. Microbiol. 38:4663-4664.

11. Richter, E., S. Rüsch-Gerdes, and D. Hillemann. 2006. Evaluation of the GenoType mycobacterium assay for identification of mycobacterial species from cultures. J. Clin. Microbiol. 44:1769-1775.

12. Rüsch-Gerdes, S., C. Domehl, G. Nardi, M.R. Gismondo, H.-M. Welscher, and G.E. Pfyffer. 1999. Multicenter evaluation of the Mycobacterial growth indicator tube for testing susceptibility of Mycobacterium tuberculosis to first-line drugs. J. Clin. Microbiol. 37:45-48. 
13. Aznar, E., D. Domingo, E. Abanades, E. García-Peñuela, and M. López-Brea. 2005. Resistencia en Mycobacteriumtuberculosis durante un periodo de cuatro años en un hospital de Madrid. Rev. Esp. Quimioterapia. 18:222-225.

AU4 14. Aragó Galindo, M., A. Belda Mira, A. Albert Contell, B. Serra Sanchis, I. Amorós Quiles, J. Prat Fornells, R. Escorns Trullenque, and C. González. 2006. Resistencias de Mycobacterium tuberculosis en el área del Hospital de Sagunto desde 1999 al 2004. Rev Clin. Esp. 206:376-381.

15. Remacha Esteras, M.A., I. P Parra, A. Esteban Martín and M.D. Blanco Mercadé. 2003. Resistencias de Mariobacterium tuberculosis en un hospital de León D v v. Esp. Quimioterap. 16:455-456.

16. Álvarez, D., J.M. Valle, M.V. Martino, P. Gordo, J. Carreira, and L. Valdés. 1994. Estudio de las resistencias primarias a fármacos antituberculosos en Galicia. $\Delta$ rchh. Bronconeumol. 30:297-300.

17. Alberte -Castiñeiras, A., A. Campos-Bueno, L. LopezUrrutia, E. Alvarez-Alonso, G. Megías, E. Ojeda-Fernandez, R. Lopez-Medrano, J. Iglesias García, S. García-Carbajosa, and P. Pérez-Pascual. 2010. Resistencias a fármacos de Mycobacterium tuberculosis en la Comunidad de Castilla y (España), 2001-2005: tercer estudio multicéntrico. 20 rm. Infecc. Microbiol. Clin. 28:706-709.

18. Casal, M., J.B. Gutiérrez, and P. Ruiz. 2000. Estado actual de la resistencia múltiple a fármacos en la tuberculosis. (2) Esp. Quimioterap. 13:167-170.

19. Idigoras, P., A. Valiente, L. Iglesias, L. Piñeiro, and E. Pérez-Trallero. 2002. Bajo nivel de resistencia a fármacos en cepas de Mycobactarium tuberculosis aisladas en Gipuzkoa (1993-2001). EDerm. Infecc. Microbiol. Clin 20: $187-188$.

20. Jiménez, M.S., and M. Casal. 2008. Situación de las resistenciz fármacos de Mycobacterium tuberculosis en España.Q. Esp. Quimioterapia. 21:22-25.

21. Marín Royo,M., F. González Morán, R. Moreno Muñoz, F. Pardo Serrano, P. Prada Alfaro, A. Arnedo Pena, and M.C. Aguar Benito. 2000. Evolución de las resistencias a My22 cobacterium tuber is en la provincia de Castellón. Años 1992-1998. B. Bronconeumol. 36:551-556.

AU5 2. Martín-Casabona, N., F. Alcaide, P. Coll, J. González, J.M. Manterola, M. Salvadó, and J.A. Caylà. 2000. Farmacorresistencia de Mycobacterium rculosis. Estudio multicéntrico en el área de Barcelona. Clin. 115:493-498.

3. Ruiz López, F.J., J.M. Zarauz García, M.M. Ortiz Romero, J.R. Valero Martinez, C. Peñalver Mellado, F. Sánchez Gascón, and M. Lorenzo Cruz. 2006. merculosis en la comarca de Lorca: adaptarse o resistir $\Omega$ Med. Interna. 23:321-325.

24. Sanz Barbero B., and T. Blasco Hernández. 2007. Situación actual de las resistencias de Mycobacterium tuberculpcicen la población inmigrante de la Comunidad de Madrid $\triangle$ h. Bronconeomol. 43:324-333.

25. Tirado Balaguer, M.D., R. Moreno Muñoz, M. Marín Royo, F. González Morán, F. Pardo Serrano, A. García del Busto Remón, and P. Prada Alfaro. 2006. Impacto de la in- migración en las resistencias de Mycobacter $($ tuberculosis en la provincia de Castellón: 1995-2000 d. Clin. 126:761-764.

26. Torres, L., P. Arazo, J. Blas Pérez, M.P. Amador, M.A. Lezcano, M.J. Revilla, and J.B. García-Moya. 2000. Resistencia de Mycobacterium tu $C$ losis en Zaragoza (1993-1997) y factores asociados. Clin. 115:605-609.

27. Blanquer, R., T. Rodrigo, M. Casals, J. Ruiz Manzano, J.M. García-García, J.L. Calpe, E. Valencia, T. Pascual, I. Mir, M.A. Jiménez, F. Cañas, R. Vidal, R.A. Penas, and J.A. Caylà. 2015. Resistencia a fármacos antituberculosos de primera línea en España durante 2010-2011. Estudio RETUBES. Br. Bronconeumol. 51:24-30.

28. Caminal Niontero, L., L. Trapiella Martínez, J. Fernández \AU6 Bernaldo De Quirós, and M. Telenti Asensio. 2002. Características de la tuberculosis en un hospital general durante los años 1993-1998 Análisis de las resistencias y coinfección por el VIH. $\cap$ rm. Infecc. Microbiol. Clin. 20:68-73.

29. Perdigão, J., R. Mracedo, C. Silva, C. Pinto, C. Furtado, L. Brum, and I. Portugal. 2010. Tuberculosis drug-resistance in Lisbon, Portugal: a 6-year overview. Clin. Microbiol. Infect. 17:1397-1402.

30. Furtado, C., and L. Brum. 2003. Laboratory surveillance of drug resistance tuberculosis in Portugal in 2000-2001. Rev. Port Pneumol. 9:279-291.

31. Gomes, C., B. Mendes, J. Pina, and M.J. Marques Gomes.1998. Resistência aos antibacilares. Rev Port Pneumol. 4:155-163.

32. Ruiz, P., J.B. Gutiérrez, F. Rodríguez-Cano, F.J. Zerolo, and M. Casal. 2004. Activity of Rifampin against Mycobacterium tuberculosis in a Reference Center. Microb. Drug Resist. 10:239-242.

33. Ruiz P, F. Rodríguez-Cano, F.J. Zerolo, and M. Casal. 2003. Current interest of isoniazid in the chemotherapy of tuberculosis in the light of its in vitro activity. Microb. Drug Resist. Fall. 9:313-316.

34. Ruiz P, F. Rodríguez-Cano, F.J. Zerolo, and M. Casal. 2002. Investigation of the in vitro activity of streptomycin against Mycobacterium tuberculosis. Microb. Drug Resist. 8:147-149.

35. Gilpin, C, A. Korobitsyn, and K. Weyer. 2016. Current tools available for the diagnosis of drug-resistant tuberculosis. Ther. Adv. Infect. Dis. 3:145-151.

Address correspondence to: Manuel Casal $\bigcirc 7$

Facultad de Medicina

Centro de Referencia para el control de tuberculosis Servicio de Microbiología Hospital

Universitario Reina Sofía Avda Menendez Pidal S/N Córdoba 14004 Spain

E-mail:mi1carom@uco.es 


\section{AUTHOR QUERY FOR MDR-2017-0353-VER9-GUTIERREZ-AROCA_1P}

AU1: Please note that gene symbols in any article should be formatted as per the gene nomenclature. Thus, please make sure that gene symbols, if any in this article, are italicized.

AU2: Please review all authors' surnames for accurate indexing citations.

AU3: Please confirm the correctness of authors' affiliation.

AU4: In Refs. 14, 15, 17-28, 31 are in other language. Please provide in English.

AU5: "Ref. 23" number is repeated twice in reference list for two different reference, so we did not renumber the references. Please check and fix.

AU6: Please cite Ref. 28 in text.

AU7: Please mention the degrees for corresponding author.

AU8: Please mention the significance of bold in Table 1 . 